\title{
Does Type 2 Diabetes Mellitus Affects Bone Mineral Density?
}

\section{Payam Sarmadi 1, ${ }^{凶}$, Behnaz Bazrafshan ${ }^{2}$, Hamidreza Bazrafshan ${ }^{1,3}$, G.K. Stalla ${ }^{3}$, Mehrdad Aghaei ${ }^{4}$, Hossien} Shadpourdashti ${ }^{4}$, Fatemeh Mousavi ${ }^{1}$, Mamak Eftekharie ${ }^{1}$

\begin{abstract}
1) Toloo Endocrine clinic, Tehran, Iran with cooperation of Max Planck Institute Munich, Germany
2) Medical Celfular and Molecular Research Center, Golestan University of Medical Sciences, Gorgan, Iran

3) Neuroendocrinology Group, Max Planck Institute of Psychiatry, Munich, Germany

4) Joint, bone \& Connective Tissue Research Center, Golestan University of Medical Sciences, Gorgan, Iran

凶: sarmadi_payam@yahoo.com
\end{abstract}

Introduction: The effects of Diabetes Mellitus (DM) on bone mineralization and osteoporosis is not well established. While reduction in bone mineral density in patients with type 1 diabetes is almost confirmed, effects of type 2 diabetes on bone health is still unclear. However in some studies Bone Mineral Density (BMD) was suggested to be normal or elevated, epidemiological reports have shown an increased risk of fracture among patients with type 2 diabetes. This paper tries to report interim results of the study which was designed to evaluate the probable association between type 2 diabetes and osteoporosis.

Method: In this study, 1000 subject were selected randomly from patients undergoing bone densitometry in Gorgan, Iran. Their demographic data, history of concomitant disease and history of pharmacologic treatment were extracted. Bone mineral density was assessed by Norland machine at the lumbar and femoral neck. Data of patients with type 2 diabetes were separated. All data were analyzed by SPSS software version 16, using T-test.
Table 1. Mean T score, BMD and Prevalence of Osteoporosis in diabetic patients

\begin{tabular}{|c|c|c|c|}
\hline & T-Score & BMD value & $\begin{array}{c}\text { Prevalence Of } \\
\text { Osteoporosis }\end{array}$ \\
\hline Lumbar Spine & -0.60 & 0.90 & $31.06 \%$ \\
\hline Femoral Neck & -1.48 & 0.75 & $38.96 \%$ \\
\hline
\end{tabular}

Results: From the 1000 studied patients, 367 (36.7\%) were diabetic. The mean age of diabetic patients was 56.23 years. $325(88.55 \%)$ of these patients were female. In diabetic patients, mean T-Score at lumbar spine and femoral neck were $-0.60 \pm 7.11$ and $-1.48 \pm 6.80$ respectively. Mean BMD value at lumbar spine and femoral neck were also $0.90 \pm$ 0.23 and $0.75 \pm 0.13$ respectively. The prevalence of osteoporosis at lumbar spine and femoral neck of diabetic patients were $31.06 \%$ and $38.96 \%$, respectively. The association between DM with BMD and Tscore in the femoral and lumbar areas was not statistically significant. $(\mathrm{P}$-value $>0.05)$.

Conclusion: According to our results, it seems that type 2 diabetes mellitus does not affect bone density and the causes of increased risk of fracture in dieses patients can be other complications of DM (such as sensory neuropathy or visual impairment) however further studies are recommended. 